Revista Brasileira de Agricultura Irrigada v.11, nº.8, p. 2152 - 2161, 2017

ISSN 1982-7679 (On-line)

Fortaleza, CE, INOVAGRI - http://www.inovagri.org.br

DOI: $10.7127 /$ rbai.v11n800704

Protocolo 704.17 - 23/06/2017 Aprovado em 08/09/2017

\title{
AJUSTE DOS PARÂMETROS DA EQUAÇÃO HARGREAVES-SAMANI EM ESCALA DIÁRIA PARA O PERÍMETRO IRRIGADO JACARÉ-CURITUBA, CANINDÉ-SE
}

Allan Cunha Barros ${ }^{1}$, Carlos Sérgio de Oliveira Silva ${ }^{2}$, Antenor de Oliveira Aguiar Netto $^{3}$

\begin{abstract}
RESUMO
A baixa disponibilidade de dados climatológicos torna o uso da equação de Hargreaves e Samani (1985) - HS uma alternativa na estimativa dos valores de ETo, possibilitando o aumento da qualidade da gestão da água, em especial nos perímetros irrigados, como o Jacaré-Curituba, localizado no Sertão Sergipano. Apesar da simplicidade, alguns autores recomendam o ajuste dos parâmetros da equação de HS, para a redução dos erros da estimativa. Assim, o objetivo deste trabalho foi ajustar os parâmetros da equação de HS para seu uso no perímetro irrigado Jacaré-Curituba-SE. O projeto foi feito na Universidade Federal de Alagoas, em parceria com a Universidade Federal de Sergipe. Utilizou-se uma séria de dados de 2006 a 2013 e os tratamentos foram baseados no ajuste de 1uma variável (HS-1), duas variáveis (HS-2), e três (HS-3). Para o ajuste da variável adotou-se a metodologia descrita por Wraith e Or (1998), em que através da ferramenta solver, eram atribuídos valores as variáveis analisadas até que o erro de estimativa dos dados de ETo estimados pelo HS, em relação ao modelo padrão PenmanMonteith (PM), fosse o menor possível. A qualidade dos ajustes foi analisada por coeficientes estatísticos. De posse dos resultados observou-se uma redução dos erros de estimativa e aumento dos índices de avaliação da qualidade das calibrações. O ajuste com maior número de variáveis ajustadas proporcionou melhores resultados, sendo suas variáveis ajustadas: "a" 0,0039, "b" 0,6397 e "c" -6,579.
\end{abstract}

Palavras-chave: Penman-Monteith, Temperatura do ar, evapotranspiração

\section{ADJUSTMENT OF HARGREAVES-SAMANI EQUATION PARAMETERS ON DAILY SCALE FOR IRRIGATED PERIMETER JACARÉ-CURITUBA, CANINDÉ- SE}

\footnotetext{
ABSTRACT

${ }^{1}$ Eng. Agrônomo. Professor do Curso de Agronomia da UFAL, Campus Arapiraca, Av. Manoel Severino Barbosa, Bom Sucesso, Arapiraca, AL, Brasil. email: allan.cunha.barros@gmail.com ${ }^{2}$ Estudante de Eng. Civil da Ufal, Campus Sertão, Rodovia AL 145, Km 3, n 3849, Delmiro Gouveia, AL, Brasil. email: carlosergio.ufal@gmail.com

${ }^{3}$ Eng. Agrônomo. Professor do Curso de Eng. Agronômica da UFS, Departamento de Eng. Agronômica, Av. Marechal Rondon, S/n - Jardim Rosa Elze, São Cristóvão, SE, Brasil. email: antenor.ufs@ gmail.com
} 
The low availability of climatological data makes the use of the equation of Hargreaves and Samani (1985) - HS an alternative in the estimation of ETo values, making possible an increase in the quality of water management, especially in irrigated perimeters such as Jacaré-Curituba, located in the Sergipe. Despite the simplicity, some authors recommend adjusting the parameters of the HS equation to reduce the estimation errors. Thus, the objective of this work was to adjust the parameters of the HS equation for its use in the Jacare-Curituba-SE irrigated perimeter. The project was done at the Federal University of Alagoas, in partnership with the Federal University of Sergipe. A series of data from 2006 to 2013 was used and the treatments were based on the adjustment of one variable (HS-1), two variables (HS-2), and three (HS-3). For the adjustment of the variable, the methodology described by Wraith and Or (1998) was adopted, in which, through the solver tool, the analyzed variables were assigned values until the estimation error of the ETo data estimated by the HS, in relation to the model Standard Penman-Monteith (PM), was the smallest possible. The quality of the adjustments was analyzed by statistical coefficients. The results obtained showed a reduction in the estimation errors and an increase in the calibration quality evaluation indices. The adjustment with the highest number of adjusted variables provided better results, and their variables were adjusted: "a" 0.0039, "b" 0.6397 and "c" -6.579.

Keywords: Penman-Monteith, Air temperature, evapotranspiration

\section{INTRODUÇÃO}

Em seu artigo de 1985, Hargreaves e Samani informavam que os principais problemas associados à estimativa da ETo era a falta de dados climatológicos e a necessidade de calibração, por isso, os mesmos propuseram um novo, para a época, modelo que teria simplicidade e praticidade, em que necessitasse o mínimo de dados climatológicos. Os mesmo ainda informavam que o modelo poderia ser utilizado sem ajuste, ou com o mínimo ajuste possível.

A metodologia de estimativa da evapotranspiração, via modelo PenmanMonteith (Allen, et al. 1998), que apesar de ser possível de ser utilizado em diversas regiões e em diversas escalas temporais, tornou o modelo como o indicado pela FAO, embora, segundo Ravazzani et al, (2012), sejam necessários uma gama de dados, dentre eles, Temperatura, radiação solar, umidade reativa, e velocidade do vento. Este tipo de modelo faz com que o equipamento, a estação meteorológica, necessite de uma gama de equipamentos, agregando valor ao equipamento, tornando de difícil acesso do ponto de vista financeiro, quando o sistema se torna automatizado, o custo se torna ainda mais elevado, fazendo com que as estações só sejam adquiridas, em sua maioria, por instituições de pesquisas e grandes produtores.

Na contra mão do modelo PM, dados para a estimativa da ETo por HS podem ser obtido com um termohigrômetro, equipamento com custo muito baixo que pode ser adquirido por qualquer produtor. Apesar disso, a utilização de um ajuste local dos parâmetros da equação faz-se necessário para que a estimativa contenha menos erros e possa ser utilizada com mais confiança. Autores como Fernandes et al. (2011), Macêdo et al. (2017), Arraes et al. (2016), Lima Júnior et al. (2016) já desenvolveram ajustes para a equação de HS possibilitando seu uso em algumas regiões do Brasil.

A falta de dados climatológicos completos e confiáveis ainda é uma verdade na agricultura brasileira, e a possibilidade de gerar estes dados é uma ferramenta importante na gestão da água nas propriedades agrícolas, em especial nos perímetros irrigados. O Perímetro Irrigado Jacaré Curituba, localiza-se a noroeste do Estado de Sergipe, na região do baixo São Francisco, ocupando uma área irrigável de 3.105 ha entre os municípios de Canindé de São Francisco e Poço Redondo (CODEVASF, 2011). Segundo Fernandes et 
al. (2011) o manejo da água e a produtividade das culturas podem ser melhorados em função da confiabilidade dos métodos utilizados para estimar a evapotranspiração das culturas. Além disso, compreender a ETo é essencial para um planejamento econômico do uso de recursos hídricos.

Pensando num uso racional dos recursos hídricos, a redução dos erros e na disponibilidade de valores de consumo hídrico de referência, através de equipamento de baixo custo, que necessite apenas valores de Temperatura, objetivou-se ajustar os parâmetros da equação de HS para seu uso no perímetro irrigado Jacaré-Curituba-, localizado na cidade de Canindé-SE.

\section{MATERIAL E MÉTODO}

O presente trabalho foi desenvolvido na Universidade Federal de Alagoas, em parceria com a Universidade Federal de Sergipe. O Perímetro Irrigado Jacaré Curituba está localizado no baixo São Francisco, cujas coordenadas geográficas são $9^{\circ} 42^{\prime} \mathrm{S}$ de latitude e $37^{\circ} 44^{\prime} \mathrm{W}$ de Longitude. Segundo classificação Köppen, a região possui clima do tipo Bssh, clima muito quente, semiárido, com 7 a 8 meses seco. Os tipos de solos predominantes são Luvissolos, Planossolos, Vertissolos e Neossolos (CODEVASF, 2011).

Os dados relativos às variáveis climatológicas como temperaturas máxima, mínima e média, umidade relativa do ar, velocidade do vento e radiação solar incidente, foram obtidos através do sítio: www.globalweather.tamu.edu do The National Centers for Environmental Prediction (NCEP). A série de dados foi obtida dos anos de 2006 a 2013, registrados em planilha eletrônica.

Utilizou-se o modelo PenmanMonteith (PM) como padrão, atendendo a recomendação da FAO, que informa a aplicabilidade do modelo para diferentes escalas de tempo e variação climática ao redor do mundo (Allen et al., 1998).

$$
\text { ETo }=\frac{0,408 \Delta\left(R_{n}-G\right)+\gamma\left(\frac{900 \times U_{2}}{T+273}\right) \times\left(e_{s}-e_{a}\right)}{\Delta+\gamma\left(1+0,34 U_{2}\right)}
$$

em que:

ETo(PM) - evapotranspiração de referência pelo método de $\mathrm{PM}$, em gramado, $\mathrm{mm} \mathrm{d}^{-1}$;

$\mathrm{Rn}$ - radiação líquida, $\mathrm{MJ} \mathrm{m}^{-2} \mathrm{~d}^{-1}$;

$\mathrm{G}$ - fluxo de calor no solo, $\mathrm{MJ} \mathrm{m}^{-2} \mathrm{~d}^{-1}$;

$\mathrm{T}$ - temperatura média do ar, ${ }^{\circ} \mathrm{C}$;

$\mathrm{V}$ - velocidade média do vento a $2 \mathrm{~m}$ de altura, $\mathrm{m} \mathrm{s}^{-1}$;

$\left(\mathrm{e}_{\mathrm{s}}-\mathrm{e}_{\mathrm{a}}\right)$ - déficit de pressão de vapor, $\mathrm{kPa}$;

$\Delta$ - curva de pressão de vapor, $\mathrm{kPa}^{\circ} \mathrm{C}^{-1}$;

$\gamma$ - constante psicrométrica, $\mathrm{kPa}^{\circ} \mathrm{C}^{-1}$, e 900 fator de conversão.

Utilizou-se o modelo de Hagreaves e Samani, proposto em 1985. Dado pela equação abaixo:

$$
\text { ETo }=a \times \frac{R a}{2,45} \times\left(T_{\max }-T_{\min }\right)^{b} \times\left(T_{\text {média }}+c\right)
$$

em que:

ETo - Evapotranspiração, mm dia- ${ }^{1}$;

$\mathrm{R}_{\mathrm{A}}$ - radiação no topo da atmosfera, $\mathrm{MJ} \mathrm{m}^{-2}$ $\mathrm{s}^{-1}$;

$\mathrm{T}_{\max }$ - temperatura máxima do ar, ${ }^{\circ} \mathrm{C}$;

$\mathrm{T}_{\min }$ - temperatura mínima do ar, ${ }^{\circ} \mathrm{C}$;

$\mathrm{T}_{\text {média }}$ - temperaturas média do ar, ${ }^{\circ} \mathrm{C}$;

Os valores de (a), (b) e (c) são os parâmetros de ajustes da equação original proposta por Hargreaves e Samani (1985). O valor de "a" corresponde a 0,0023 , o de " $b$ " a 0,5 e de "c" a 17,8. O ajuste dos parâmetros foi feito através da ferramenta solver do Excel, autores como Arraes et al. (2016) e Macêdo et al. (2017) utilizaram a mesma metodologia proposta por Wraith e Or (1998), para ajuste de equação linear, apesar do exemplo utilizado em seu trabalho ter sido com solo, o princípio de funcionamento é o mesmo. A técnica para otimização assume que quanto menor for a soma dos desvios dos quadrados, mais perfeito será o ajuste dos parâmetros.

$$
\sum_{i=1}^{n}(P M-H S)^{2}=0
$$


Em que:

$\mathrm{PM}=$ Evapotranspiração de referência por Penman-Monteith, mm dia- ${ }^{-1}$;

$\mathrm{HS}=$ Evapotranspiração de referência por Hargreaves e Samani, mm dia- ${ }^{-}$;

Apesar da recomendação do uso do modelo de HS para estimativa da ETo em escala num período superior a 5 dias, adotouse o ajuste para escala diária, justificado pelo uso que o modelo teria no manejo da irrigação. Os parâmetros ajustados foram separados em 3 tratamentos: Tratamento 1 (HS-1) - somente o parâmetro "a", foi alterado; Tratamento 2 (HS-2) - os parâmetro "a" e "b", foram alterados; Tratamento 3 (HS-3) - O parâmetros "a", "b" e "c", foram alterados. Mesma metodologia adotada por Arraes et al. (2016) e Macêdo et al. (2017).

Chagas et al. (2013) analisando a ETo na cidade de rio Real na Bahia, separou os dados analisados em período seco e período úmido, no entanto, devido a baixa precipitação da região, não se fez necessário a separação.

A análise da qualidade das calibrações foi feita através do calculou do erro-padrão de estimativa (EPE), o índice de "d" de Willmott (d), coeficientes de determinação $\left(\mathrm{R}^{2}\right)$ e precisão coeficiente de correlação " $\mathrm{r}$ " e no coeficiente de confiança ou desempenho "c" introduzidos por Camargo e Sentelhas (1997).

$$
r=\sqrt{R^{2}}
$$

em que:

$\mathrm{r}$ - coeficiente de correlação; $\mathrm{R}^{2}$ - coeficiente de determinação.

$$
d=1-\left[\frac{\Sigma P_{i}-O_{i}{ }^{2}}{\Sigma\left(\left|P_{i}-O\right|+\left|O_{i}-O\right|\right)^{2}}\right]
$$

em que:

$\mathrm{Pi}$ - valor estimado;

Oi - valor observado;

$\mathrm{O}$ - média dos valores observados.

$$
c=r * d
$$

em que:

$\mathrm{r}$ - coeficiente de correlação;

$d$ - índice de Willmott.

O índice "d" é descrito em Willmott et al. (1985) e seus valores variam entre zero (nenhuma concordância) e 1 (concordância perfeita). Já o índice de desempenho "c" é descrito em Camargo e Sentelhas (1997).

\section{RESULTADOS E DISCUSSÃO}

Os dados de temperatura média de máxima, mínima, radiação solar umidade relativa do ar e precipitação podem ser vistos na Figura 1. As maiores temperaturas são encontradas nos meses de novembro à abril, com valores superiores a $35{ }^{\circ} \mathrm{C}$. O declínio das temperaturas coincidem com início da estação chuvosa que vai de março à setembro, com precipitação média anual de $315 \mathrm{~mm}$. A radiação solar possui similaridade com as curvas de temperatura, possuindo como valores máximos médios os encontrados no período de dezembro e janeiro, atingindo valores acima de $25 \mathrm{Mj} \mathrm{m}^{-2} \mathrm{Dia}^{-1}$.

A umidade relativa do ar (UR), possui valores baixos ao longo do ano, comparativamente à cidade de Maceió, AL, localizada em latitude próxima à analisada, Macêdo et al. (2017) encontraram valores de no mínimo $76 \%$. Já o perímetro irrigado Jacaré-Curituba, possui UR máxima de $76,59 \%$, encontrado no mês de julho, já a menor valor de UR foi encontrado no mês de dezembro com $54,46 \%$. 

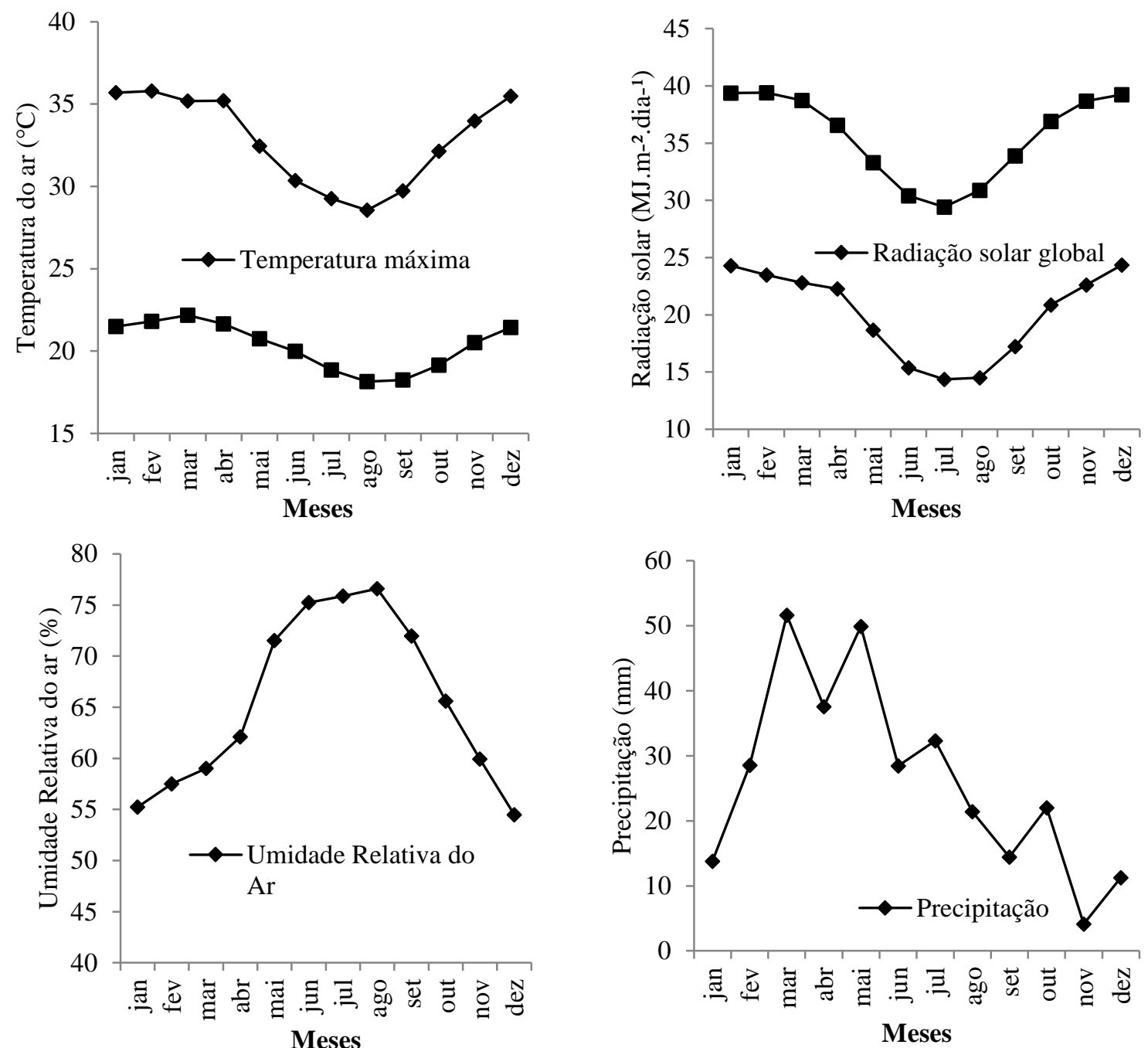

Figura 1. Valores médios mensais para os anos de 2006 a 2013 de temperatura do ar, radiação solar, umidade relativa e precipitação para o perímetro irrigado Jacaré-Curituba na cidade de Canindé-SE.

Na Figura 2 observam-se as regressões lineares com suas regressões completas e forçada pela origem. Observa-se que pelo modelo original (Figura 2 a), o coeficiente de determinação $\left(\mathrm{R}^{2}\right)$ encontrado possui valor de 0,9, demonstrando baixa dispersão dos dados, o valor encontrado foi mantido no tratamento 1 (HS-1) e somente sendo superado no tratamento 3 (HS-3), em que foram ajustados os três parâmetros da equação, apesar disso, a melhora só significou, no $\mathrm{R}^{2}$ um aumento de $3 \%$. No tratamento 3 , HS-2, houve uma queda na dispersão, com $\mathrm{R}^{2}$ em aproximadamente $0,89 \%$.

Apesar dos coeficientes $\mathrm{R}^{\mathbf{2}}$ na regressão completa apresentarem valores altos, $\mathrm{O}$ modelo HS em todos os casos apresentou uma tendência de subestimar a ETo por PM, essa subestimativa foi de $12,64 \%, 1,68 \%$, $1,33 \%$ e $1,09 \%$. Após as calibrações percebese que os valores da equação de HS se aproximam do modelo padrão, demonstrando a qualidade dos ajustes feitos até mesmo para uma única variável encontrada. Corroborando a tendência dos valores encontrados neste projeto, Arraes et al. (2016) obteve uma subestimativa do modelo HS-O em relação ao PM para cidade de Petrolina em $86,7 \%$ dos casos, valor muito alto.

Chagas et al. (2013) encontraram que o valor de HS-O subestimava o valor de PM em 20,55\%. Oliveira et al. (2005) compraram métodos de estimativa da ETo com o método padrão (Pmon) para Santo Antônio de Goiás, GO, superestimaram em $24,5 \%$. 
Barros et al.
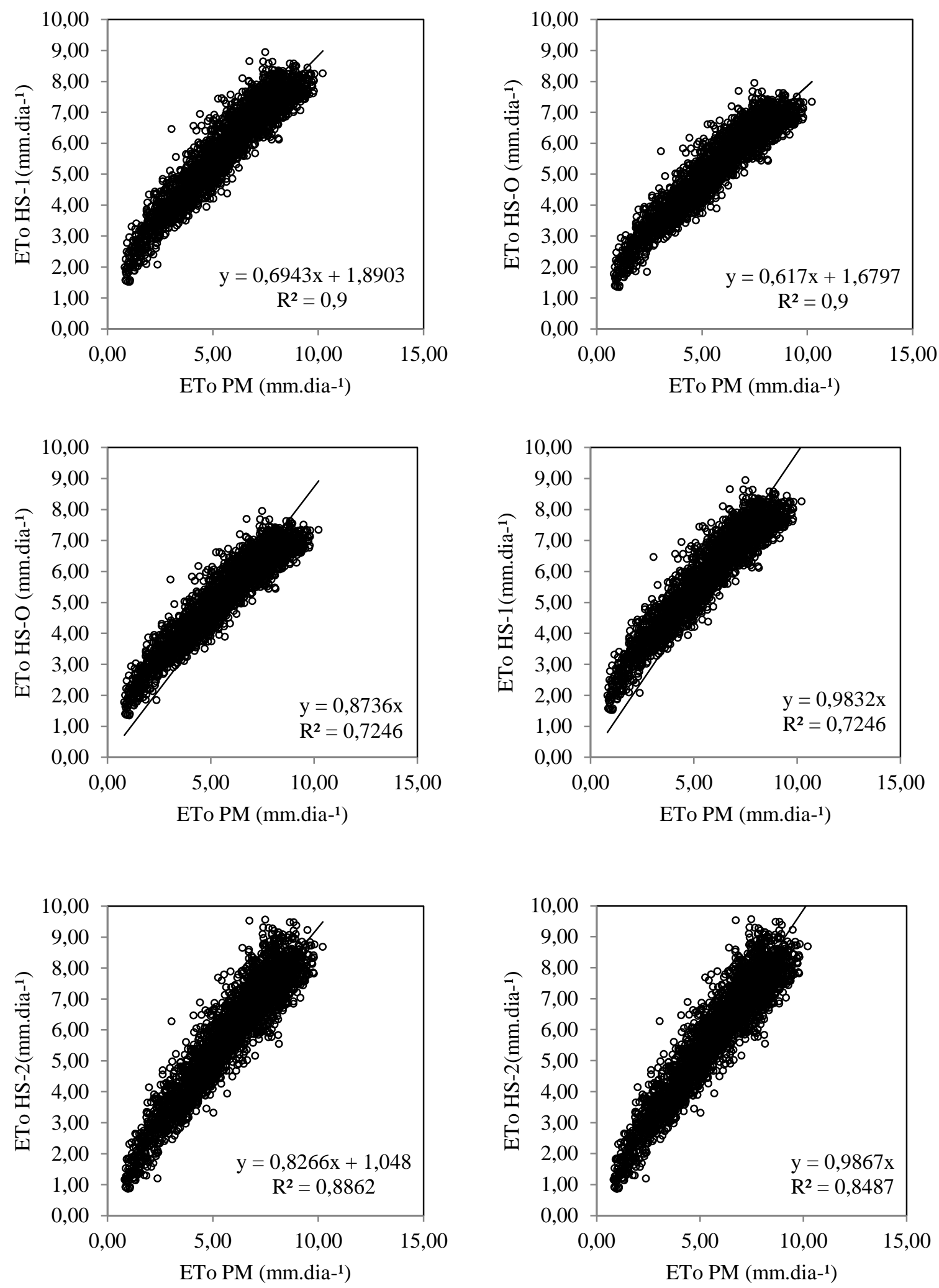

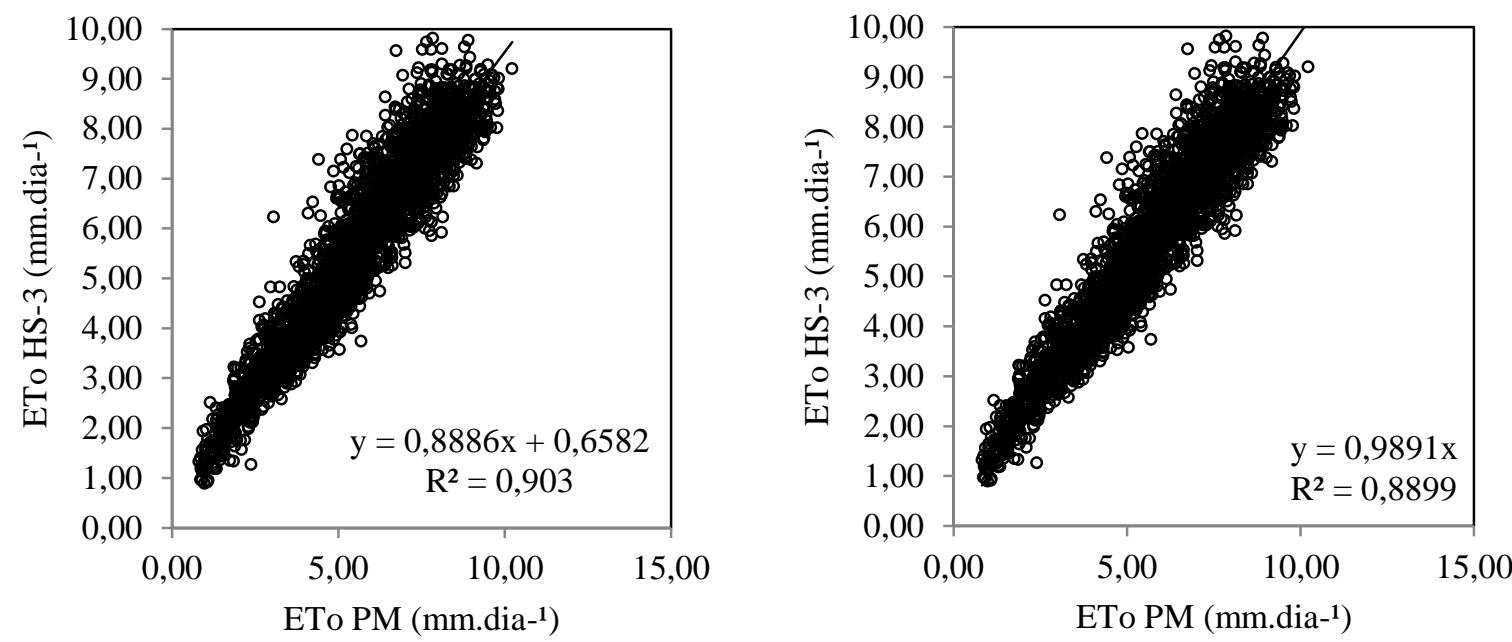

Figura 2. Regressão linear entre valores diários da evapotranspiração de referência (ETo) estimados pelo modelo de Penman-Monteith (Pmon) (eixo x) em relação ao modelo de Hargreaves-Samani original (HS0) equação completa (a) e forçada pela raiz (b), Hargreaves-Samani, com ajuste da variável a (HS-1) equação completa (c) e forçada pela raiz (d), Hargreaves-Samani, com ajuste da variável a e b (HS-2) equação completa (e) e forçada pela raiz (f) e Hargreaves-Samani, com ajuste da variável a, b e c (HS-3) equação completa $(\mathrm{g})$ e forçada pela raiz $(\mathrm{h})$.

De acordo com a Tabela 1 houve uma redução gradual do erro de estimativa à medida que ocorriam os ajustes dos parâmetros e com o número de parâmetros ajustados. O erro no modelo original foi acima de 1,00 $\mathrm{mm} \mathrm{dia-}^{-1}$, que representa um erro de $18,15 \%$ em relação à média da Eto calculada pelo modelo de PM. Apesar disso, houve uma redução do erro em 23,8\%, $32,38 \%$ e $39,05 \%$, para os tratamentos HS-1, HS-2 e HS-3, respectivamente. A concordância, "d" de Willmot, teve aumento gradual chegando a atingir o valor de 0,97, nos tratamentos HS-2 e HS-3, vale ressaltar que a concordância no modelo original já era de 0,91 .

Os coeficientes foram todos classificados como quase perfeitos, apenas o HS-2 obteve valor diferente dos demais, sendo o menor valor encontrado. O índice "c" foi classificado como ótimo e teve seu valor crescente com o aumento das variáveis ajustados do modelo. Dessa forma, é possível inferir que quanto mais coeficientes forem ajustados, melhores serão os resultados encontrados e menores serão os erros.

Chagas et al. (2013) encontraram valor de erro de $1,12 \mathrm{~mm}$ dia-$^{-1}$ avaliando o modelo HS e PM, em período seco na cidade de Rio
Real - BA, e "d" de 0,82. O índice "c" foi classificado como sofrível apesar de uma correlação muito alta.

Macêdo et al. (2017) obtiveram erro de estimativa de $0,57 \mathrm{~mm} \mathrm{dia}^{-1}$, para acidade de Maceió-AL, em comparação do modelo de HS ao de PM, e apresentaram comportamento semelhante ao encontrado neste trabalho, em que quanto maior o número de parâmetros ajustados, menor o erro e melhor a qualidade das estimativas. Apesar disso, os autores encontraram valores de concordância e desempenho menores dos que encontrados neste trabalho.

Tabela 1. Valores dos coeficientes estatísticos para análise de desempenho dos modelos original e ajustados para a equação de Hargreaves-Samani(1985) em comparação ao modelo padrão, PM para o perímetro irrigado Jacaré-Curituba na cidade de Canindé-SE.

\begin{tabular}{cccccc}
\hline \multirow{2}{*}{ Correlação } & \multicolumn{4}{c}{ Parâmetros estatísticos } \\
\cline { 3 - 6 } Original & PM & 1,05 & 0,91 & 0,95 & 0,86 \\
\cline { 3 - 6 } HS-1 & PM & 0,8 & 0,95 & 0,95 & 0,90 \\
HS-2 & PM & 0,71 & 0,97 & 0,94 & 0,91 \\
HS-3 & PM & 0,64 & 0,97 & 0,95 & 0,92 \\
\hline
\end{tabular}


Das três formas de calibração adotadas o ajuste com apenas o parâmetro " $a$ ", obteve o valor de 0,002588 (Tabela 2). De acordo com Arraes, et al. (2016) o valor de "a" encontrado para diversas cidades do estado de Pernambuco variaram de 0,00068 até 0,00241. Demonstrando a importância destes ajustes para cada localidade. Borges Júnior et al. (2012), calibraram o modelo separando seu uso de primavera-verão e de outonoinverno, na região de Garanhuns-PE.

No processo de calibração simultânea, com duas variáveis (HS-2) observa-se a redução do valor de "a" em relação ao HS-O e aumento do valor de "b". Macêdo, et al (2017) observou comportamento oposto. O mesmo aconteceu com Lima Júnior et al. (2016) que utilizando o processo de calibração simultânea, com duas variáveis, chegaram a obtiver valor praticamente nulo de 0,0004 para a cidade de Campos Sales-CE, até o valor de 0,0024 para acidade de Fortaleza -CE e houve acréscimo do parâmetro "b" em todas as condições analisadas.

Quando ajustados os três parâmetros, o parâmetro "c" obteve valor negativo. Segundo Ravazanni et al. (2012) o parâmetro "c" originalmente era utilizado para utilizado para converter a temperatura de Fahrenheit para Celsius $(32 / 1,8=17,8)$. Macêdo et al. (2017) observou o valor nulo, e informa que o mesmo possuía pouca importância para o ajuste de sua equação.

Tabela 2. Valores dos coeficientes originais e ajustados para a equação de HargreavesSamani(1983, ajustados através do Solver, para os diferentes tratamento para 0 perímetro irrigado Jacaré-Curituba na cidade de Canindé-SE.

\begin{tabular}{cccc}
\hline Tratamentos & $\mathrm{a}$ & $\mathrm{b}$ & $\mathrm{c}$ \\
\hline Original & 0,0023 & 0,5 & 17,8 \\
HS-1 & 0,002588 & & \\
HS-2 & 0,001315 & 0,7598 & \\
HS-3 & 0,0039 & 0,6392 & $-6,579$ \\
\hline
\end{tabular}

Através de análise visual dos dados de ETo estimados pelos modelos de PM, HS-O, HS-1, HS-2 e HS-3 é possível observar que o comportamento da ETo ao longo do ano (Figura 3). Durante os meses com baixa radiação, global, de maio a setembro, os modelos HS-O e HS-3 possuem valores quase iguais aos do modelo PM. PM só é subestimado pelo modelo HS-3, no período de fevereiro a abril. O mês com maior discrepância entre o modelo PM e os outros foi o de dezembro. O modelo que obteve os valores mais próximos do PM foi o HS-3. Embora todos os modelos avaliados apresentaram comportamento semelhante ao PM.

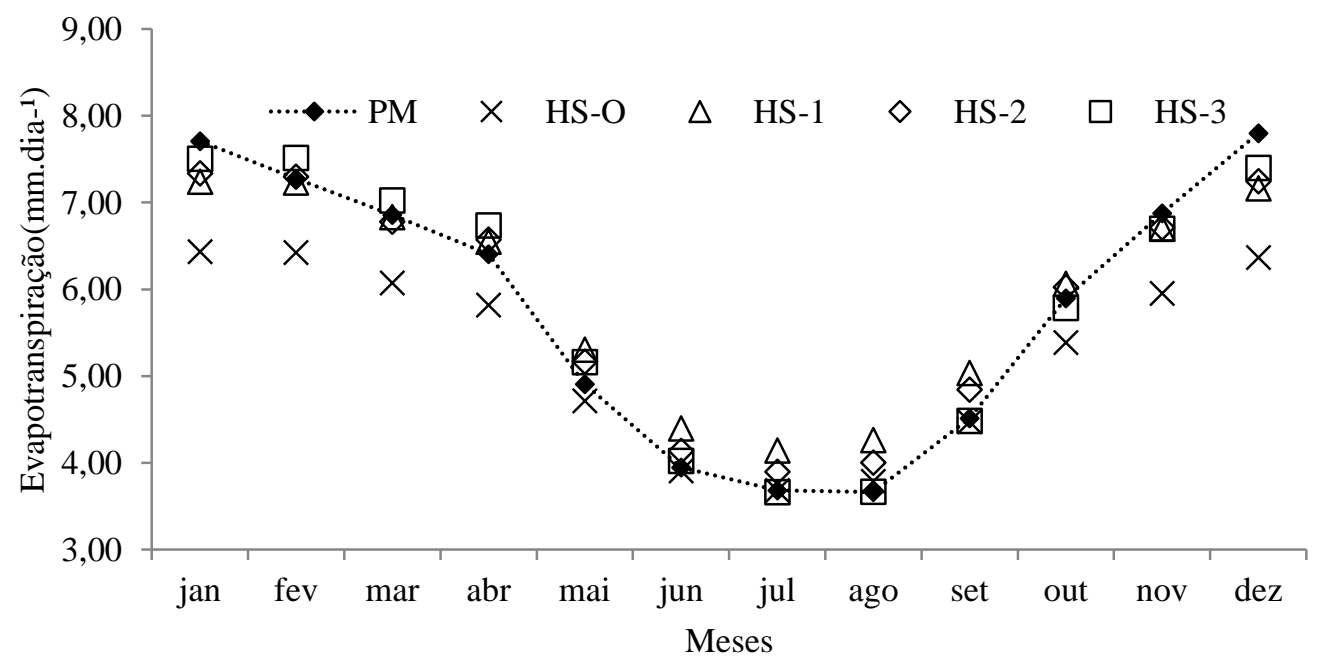

Figura 3. Variação média mensal dos valores de Evapotranspiração de referência pelos modelos de Penman-Monteith (PM), Hargreaves e Samani com o ajuste original ( HS-O), Hargreaves e Samani com o ajuste no parâmetro "a" (HS-1), Hargreaves e Samani com o ajuste no parâmetro "a" e "b" (HS-2) e Hargreaves e Samani com o ajuste no parâmetro "a", "b" e "c" (HS-3). 


\section{CONCLUSÃO}

Foi possível ajustar os parâmetros do modelo HS para seu uso em escala diária no perímetro irrigado Jacaré-Curituba, localizado na cidade de Canindé-SE.

Todas os tratamentos de ajustes apresentaram valores de erro inferiores ao do modelo original, proporcionando aumento da confiabilidade

O ajuste com maior número de variáveis ajustadas proporcionou melhores resultados, sendo suas variáveis ajustadas: "a" 0,0039, "b" 0,6397 e "c" 6,579, reduzindo o erro de estimativa em $39,05 \%$.

\section{REFERÊNCIA BIBLIOGRÁFICA}

ALLEN, R. G. et al. Crop evapotranspiration: guidelines fo computing crop water requirements. Irrigation and Drainage Paper 56. p. 300, 1998.

ARRAES, F. D. D. LIMA JUNIOR, J. C. OLIVEIRA, J. B. MACÊDO, K. G. COURAS, Y. S. OLIVEIRA, W. C. Parametrização da equação de HargreavesSamani para o estado do Pernambuco - Brasil. Revista Brasileira de Agricultura Irrigada, v. 10, n. 1, p. 410419, 2016.

BORGES JÚNIOR, J. C. F., ANJOS, R. J., SILVA, T. J. A., LIMA, J. R. S., ANDRADE,C. L. T. Métodos de estimativa da evapotranspiração de referência diária para a microrregião de Garanhuns, PE. Revista Brasileira de Engenharia Agrícola e Ambiental, v. 16, n. 4, p. 380-390, 2012.

CAMARGO, A.P.; SENTELHAS, P.C. Avaliação do desempenho de diferentes métodos de estimativa da evapotranspiração potencial no Estado de São Paulo, Brasil. Rev. Bras. Agrometeorologia, Santa Maria, v. 5, n. 1, p. 89-97, 1997.
CHAGAS, R. M.; FACCIOLI, G. G.; AGUIAR NETTO, A. O.; SOUSA, I. F.; VASCO, A. N.; SILVA, M. G. Comparação entre métodos de estimativa da evapotranspiração de referência (ETo) no município de Rio Real-BA. Irriga, Botucatu, v. 18, n. 1, p. 351-363, 2013.

CODEVASF, Perímetros Irrigados: Jacaré-Curituba, 2011.

FERNANDES, D. S., HEINEMANN, A. B., PAZ, R. L. F. OLIVEIRA, A. A. Calibração regional e local da equação de Hargreaves para estimativa da evapotranspiração de referência. Revista Ciência Agronômica, v. 43, n. 2, p. 246-255, 2011.

HARGREAVES, G. H.; SAMANI, Z. A. Reference crop evapotranspiration from temperature. Applied Engineering in Agriculture, v. 01, n. 02, p. 96-99, 1985.

LIMA JUNIOR, J. C., ARRAES, F. D. D., OLIVEIRA, J. B. D., NASCIMENTO, F. A. L. D., MACÊDO, K. G. Defining parameters for the Hargreaves-Samani equation for estimating reference evapotranspiration in the State of Ceará, Brazil. Revista Ciência Agronômica, v. 47, n. 3, p. 447-454, 2016.

MACÊDO, K. A.; ARRAES, F. D. D. ; LIMA JUNIOR, J. C. ,; OLIVEIRA, W.C. ,; ARAUJO, Y. R.; Ajuste dos parâmetros do modelo de Hargreaves E Samani para estimativa da evapotranspiração de referência em escala diária para Maceió-AL.

Revista Brasileira de Agricultura Irrigada, v.11, no.3, p. 1484 - 1491, 2017.

OLIVEIRA, R. Z.; OLIVEIRA, L. F. C.; WEHY, T. R.; BORGES, L. B.; BONONO, R. Comparação de metodologias de estimativa da evapotranspiração de referência para a região de Goiânia, GO. Biosci. J., v.21, n.3, p.19-27, 2005.

RAVAZZANI, G., CORBARI, C., MORELLA, S., GIANOLI, P., \& MANCINI, 
Barros et al.

M. Modified HargreavesSamani equation for the assessment of reference evapotranspiration in Alpine river basins. Journal of Irrigation and Drainage Engineering, v. 138, n. 7, p. 592-599, 2011.
WRAITH, J. M.; OR, D. Nonlinear parameter estimation using spreadsheet software. Journal of Natural Resources and Life Sciences Education, v. 27, p. 1319, 1998. 